\title{
Registration of observational studies: Is it time?
}

\author{
Rebecca J. Williams PharmD MPH, Tony Tse PhD, William R. Harlan MD, Deborah A. Zarin MD
}

Previously published at www.cmaj.ca

$\mathrm{O}$ bservational studies form an important part of the medical evidence base, particularly for assessing rare adverse events and long-term effectiveness of medications and devices. ${ }^{1}$ However, observational studies, like interventional studies (clinical trials), are subject to publication bias and reporting bias. ${ }^{2-4}$ Registration of clinical trials is a widely recognized tool for facilitating complete public reporting. ${ }^{5}$ Registration of observational studies has received less attention, although interest is growing. ${ }^{6-8}$ Because existing registries (e.g., ClinicalTrials.gov) accommodate observational studies, and the rationale and benefits of registration are similar, we ask the scientific community and other stakeholders to consider the systematic, prospective registration of observational studies.

\section{Why register observational studies?}

Much of the rationale for the prospective registration of clinical trials ${ }^{9}$ applies to the registration of observational studies (Table 1). ${ }^{7}$ For example, observational studies in which researchers acquire data directly from human participants entail ethical obligations to participants, even though such research generally involves less risk than interventional studies. ${ }^{10}$ These obligations include oversight by ethical review boards, informed consent, and public release of the study findings to advance biomedical knowledge. As with clinical trials, incomplete reporting of observational studies has been documented. ${ }^{3}$ Some researchers suggest that observational studies are also at increased risk for publication bias or other types of bias, including misrepresentation of prespecified analyses or phenotype definitions. ${ }^{2,4}$ Such biases are a concern because they undermine the validity of observational studies, which are an important component of the medical evidence base in areas of public health, such as detection of rare adverse events. ${ }^{1.16}$

Observational studies of medications and devices are playing a more visible role at the United States Food and Drug Administration (FDA) (Table 2). For instance, the FDA posted an "Early communication about an ongoing safety review"18 in response to a published observational study associating abacavir and didanosine with an increased risk of cardiovascular and cerebrovascular events. ${ }^{19}$ The authors of the study noted that, although a randomized controlled trial is necessary to show a causal association, such a trial design is "unlikely to be feasible," given that it would require more than 10000 participants to be followed for at least two years. In addition, there may be ethical concerns in conducting a randomized controlled trial if harms are expected. Given that this and other associations between marketed products and possible harms are likely to be investigated further using observational stud-

\section{Key points}

- Clinical trial registries are established tools for improving access to information on trials and for addressing publication bias and reporting bias.

- Much of the ethical and scientific rationale for registering clinical trials also applies to observational studies.

- The existing infrastructure for trial registration is being used for observational studies, which make up $17 \%$ of the studies registered in ClinicalTrials.gov.

- Further discussion is necessary to assess the scope and specific implementation-related issues of systematic registration of observational studies.

ies, a registry containing summary protocol information would allow researchers to track such studies from initiation to completion. Such a tool could be useful to researchers who are evaluating the current evidence, considering initiating similar studies, identifying gaps in research or seeking collaborators. ${ }^{15}$ Similarly, a database of summary results could improve access to information about published and unpublished observational study analyses (whether prespecified or post hoc), thereby mitigating publication bias and incomplete reporting of results.

Given these potential benefits, observational studies are already being registered for various reasons, and there is increasing attention given to this practice. For example, the state of Maine requires registration and reporting of results of postmarketing observational studies of medications and biological products marketed in Maine ${ }^{20}$ and the corporate policies of some drug manufacturers address disclosure of observational studies. ${ }^{21}$ Recently, an international workshop was held on the topic, ${ }^{6}$ and several medical journals published editorials on the registration of observational studies. ${ }^{7,822}$ Some have suggested that ethics review boards should require prospective registration of any study involving human participants, ${ }^{12}$ whereas others have proposed that observational studies under the mandate of the FDA should be subject to the same requirements for registration and reporting of results as those for clinical trials. ${ }^{17,23}$ The European Medicines Agency recently issued a work plan to create a registry of post-authorization safety studies that would include observational studies, ${ }^{24}$ and a draft report by the Agency for Healthcare Research and Quality considered the utility of creating a "registry of

From the National Library of Medicine, National Institutes of Health, United States Department of Health and Human Services, Bethesda, USA

CMAJ 2010. DOI:10.1503/cmaj.092252 
patient registries. ${ }^{25}$ With growing interest in this topic, an understanding of current practices in registration is useful.

\section{Where can observational studies be registered?}

An increasing number of clinical trial registries exist and, despite their names, some include observational studies. For example, ClinicalTrials.gov (http://clinicaltrials.gov), established by the US National Library of Medicine on behalf of the US National Institutes of Health, is the largest publicly accessible registry, with over 87000 ongoing and completed interventional and observational studies as of March 2010. There are 10 registries in the World Health Organization's Registry Network, which range in size from 25 to more than 8400 registered studies as of March 2010. Of these registries, five explicitly state that observational studies can be registered (Appendix 1, available at www.cmaj.ca/cgi/content/full /cmaj.092252/DC1). A structured search tool for identifying observational studies is provided at ClinicalTrials.gov, where such studies represent $17 \%(n=14595)$ of all registrations, and at the Australian New Zealand Clinical Trials Registry, where $5 \%(n=214)$ of registered studies are observational.

Table 1: Rationale for registration of clinical trials and application to observational studies

Rationale for registration of clinical trials

Application to observational studies

Respect for human

participants

Evidence-based medicine

Mitigation of publication

bias and detection of

deviations

Clear documentation of prespecified study design

Identification of gaps

in research

Avoidance of duplication

Public record
Observational studies involve human participants and thereby entail ethical obligations. ${ }^{10}$ Individuals deserve to know that the study will result in information that will advance medical knowledge.

Complete set of evidence is required to practise evidence-based medicine. Observational studies are

Studies or outcomes with positive or statistically significant results may be more likely to be published (or suppressed). ${ }^{4,12,13}$ Unacknowledged changes to protocol could lead to misinterpretation of findings.

Interpretation of findings requires clear documentation of research methods. Reporting guidelines, such as STROBE (Strengthening the Reporting of Observational Studies in Epidemiology), identify protocol-related items that can be prespecified and are essential for interpreting the methods and results of an observational study. ${ }^{14}$

Gaps in evidence base are identified and opportunities for collaborative research provided. Records could signal a need for research or provide opportunities for collaborative research (e.g., assembling large cohorts to conduct genome-wide association studies). ${ }^{15}$

Support and conduct of redundant research studies are avoided. Funding through grants and allocation of limited research resources are optimized.

Registration is a permanent, public record of the study. The ClinicalTrials.gov unique identifier (NCT number) can be used in all publications and future discourse, including systematic reviews, regarding the study. considered to be part of the complete set of evidence.
Studies" from the "Study Type" dropdown menu on the advanced search form at ClinicalTrials.gov (http://clinicaltrials .gov/ct2/search/advanced).

In October 2007, modifications were made to the design-specific data elements used for registering observational studies. These changes were strongly influenced by protocol-related items in the statement Strengthening the Reporting of Observational Studies in Epidemiology (STROBE).$^{14}$ The STROBE initiative aims to improve the reporting of observational studies by specifying a core set of items to include in a publication that would allow a reader to follow "what was planned, done, and found." ${ }^{14}$ Consistent with the aims of the STROBE initiative, registration facilitates prospective documentation of key prespecified study attributes. In September 2008, ClinicalTrials.gov added a database of summary results that allows for reporting of many of the results-related items identified in the STROBE initiative, such as information on study participants (e.g., numbers of participants starting and completing the study, baseline demographic characteristics), outcome measures (including appropriate statistical analyses) and adverse events, if relevant.

After the design-specific data elements for observational studies were updated, prospective cohort studies $(n=2617)$ 
comprised the largest segment of observational studies registered in ClinicalTrials.gov between November 2007 and March $2010(n=7735)$ (Appendix 2, available at www.cmaj.ca/cgi/content/full/cmaj.092252/DC1). Trends in observational studies registered in ClinicalTrials.gov from February 2000 to March 2010 include the following:

\section{Registrations and results}

The average number of observational studies registered per year has increased consistently with overall increases in registered studies. Historically, observational studies have represented about $15 \%$ of all studies registered in any given year. At the time of writing, they represented $17 \%(n=14595)$ of all registrations of studies $(n=87530)$ and 5\% $(n=85)$ of all entries of results $(n=1578)$ in ClinicalTrials.gov.

\section{Geographic distribution}

Study locations are distributed worldwide, with over $50 \%$ in North America, 20\% in Europe and 13\% in Asia (Appendix 3, available at www.cmaj.ca/cgi/content/full/cmaj.092252/DC1).

\section{Type of sponsorship}

Most registered observational studies (85\%) are funded by non-industry sources $(24 \%$ by the National Institutes of Health and other US government agencies and $61 \%$ by academic and nonprofit organizations); the remaining $15 \%$ are sponsored by industry. In comparison, $64 \%$ of registered interventional studies are funded by non-industry sources ( $10 \%$ by the US government and $54 \%$ by academic and nonprofit organizations), and $36 \%$ are sponsored by industry (see Appendix 4, available at www.cmaj.ca/cgi/content/full/cmaj .092252/DC1).

\section{Diseases and conditions studied}

The distributions of diseases and conditions evaluated among registered observational and interventional studies were generally similar across categories of conditions as derived from the National Library of Medicine Medical Subject Headings (MeSH) controlled vocabulary (Appendix 5, available at www.cmaj.ca/cgi/content/full/cmaj.092252/DC1).

These data provide a snapshot of observational studies car-

Table 2: Examples of actions by the United Stated Food and Drug Administration (FDA) related to medications and therapeutic biological products that identify evidence from observational studies, not including analyses conducted by the FDA based on postmarketing case reports

\begin{tabular}{|c|c|c|}
\hline Action; product & Observational evidence & Nature of concern \\
\hline \multicolumn{3}{|c|}{$\begin{array}{l}\text { Early communication about an ongoing } \\
\text { safety review }\end{array}$} \\
\hline Drotrecogin alfa & Retrospective medical record review & $\begin{array}{l}\text { Risk of serious bleeding events and } \\
\text { death }\end{array}$ \\
\hline Insulin glargine & Four observational studies & Risk of cancer \\
\hline \multicolumn{3}{|l|}{$\begin{array}{l}\text { Communication about an ongoing } \\
\text { safety review }\end{array}$} \\
\hline Stimulant medications for ADHD & Case-control study & Risk of sudden death \\
\hline \multicolumn{3}{|c|}{$\begin{array}{l}\text { Change to product labelling (warning } \\
\text { and boxed warning) }\end{array}$} \\
\hline \multicolumn{3}{|c|}{$\begin{array}{l}\text { Postmarketing commitment under Section } 901 \\
\text { of the Food and Drug Administration } \\
\text { Amendments Act }{ }^{17}\end{array}$} \\
\hline Fenofibric acid, choline bitarate & Observational study & $\begin{array}{l}\text { Rhabdomyolosis necessitating } \\
\text { hospital admission in patients given a } \\
\text { fibrate in combination with a statin }\end{array}$ \\
\hline Romiplostim & Antibody registry study & $\begin{array}{l}\text { Long-term consequences of } \\
\text { neutralizing antibodies }\end{array}$ \\
\hline $\begin{array}{l}\text { Romiplostim } \\
\text { Duloxetine } \\
\text { Eltrombopag }\end{array}$ & $\begin{array}{l}\text { Prospective, observational registries } \\
\text { of exposure during pregnancy }\end{array}$ & $\begin{array}{l}\text { Pregnancy and fetal outcomes of } \\
\text { women exposed to medications } \\
\text { during pregnancy }\end{array}$ \\
\hline Rotavirus vaccine, live, oral & $\begin{array}{l}\text { Large-scale observational post- } \\
\text { licensure safety study }\end{array}$ & $\begin{array}{l}\text { Serious risk of intussusceptions and } \\
\text { other serious adverse effects }\end{array}$ \\
\hline
\end{tabular}

Note: $A D H D=$ attention-deficit hyperactivity disorder

Sources: FDA: Postmarket Requirements and Commitments (www.accessdata.fda.gov/scripts/cder/pmc/index.cfm) and Early Communications about Ongoing Safety Reviews (www.fda.gov/Drugs/DrugSafety/PostmarketDrugSafetylnformationforPatientsandProviders/DrugSafetylnformationforHeathcareProfessionals/ucm070256.htm); accessed 2009 July 21. 
ried out around the world during a 10-year period. However, our findings are not representative of all observational studies conducted during that period. In addition, the accuracy of our analysis is limited by the quality and completeness of information provided by registrants.

\section{Gaps in knowledge}

Based on our experience, we believe that the existing model for and system of registration of clinical trials can be applied to observational studies. Although ClinicalTrials.gov is able to accommodate a range of observational study designs, those that involve collection of primary, prospective data may fit this model more easily because such studies are most similar to interventional studies. In each type of study, data are generally collected prospectively from well-defined groups of individuals (e.g., exposed and nonexposed) according to a protocol. As previously noted, prospective cohort studies were the most frequently registered in ClinicalTrials.gov (Appendix 2). Whether and how other types of observational studies (e.g., retrospective data collections, secondary data analyses and evaluations of data from patient registries) should be represented in a study registry requires further consideration. For example, patient registries are established to collect a defined set of standardized variables about specific patient populations, but they may or may not have specific protocols with prespecified plans for analysis. Although hundreds of patient registries have been successfully registered (e.g., http://clinicaltrials.gov/search/term =registry), when they should be registered and which attributes should be represented could be further elucidated. The Agency for Healthcare Research and Quality is currently considering these issues. ${ }^{25}$

Other challenges related to the registration of observational studies include difficulties in delineating individual studies from a broad plan for data analysis, and whether and how sub-studies and secondary studies or analyses using the same prospective data set should be registered. In addition, data elements and definitions related to data collection for study variables,${ }^{14}$ as well as the specific structure for reporting results of observational studies, may require further consideration. For example, although the ClinicalTrials.gov registry currently collects information on outcome measures, it may also be useful to prespecify other variables (e.g., baseline characteristics) that will be used to characterize a cohort or that will be included in an analysis. ${ }^{26}$

Some have questioned the practicality of registering observational studies because of the difficulty of documenting all hypotheses and have expressed concern that prespecification of all hypotheses will inhibit exploratory research. ${ }^{13,26}$ Other approaches to publicly documenting original study design and variables already exist, such as the publication of cohort profiles in the International Journal of Epidemiology. ${ }^{27}$ Registration of observational studies may be complementary to such publications, given that the registry record is a standard format, is broadly accessible and can be updated over time and given that links or cross-references between the registry and publication could be established.

\section{Next steps: Is it time to register observational studies?}

There is interest in the prospect of registering observational studies, as reviewed earlier and as shown by the number of such studies registered at ClinicalTrials.gov. We believe that making both observational and interventional studies available in a single registry will provide researchers and others with a more comprehensive view of the growing evidence base. Based on the clinical trial experience, there is a preexisting (but evolving) model and infrastructure for registration, and study sponsors are already registering observational studies on a voluntary basis. Given that much of the ethical and scientific rationale for registration of interventional studies appears to apply to prospective observational studies, we believe that further discussion among stakeholders (e.g., researchers, regulatory authorities and the public) is warranted. Such discussion is necessary to address specific issues regarding implementation, including assessing and defining common data elements that would meet the needs of the stakeholders. In the future, the data elements for registration of observational studies in ClinicalTrials.gov could be modified to better meet these needs.

This article has been peer reviewed.

Competing interests: Rebecca Williams, Tony Tse and Deborah Zarin are employed by ClinicalTrials.gov, the registry and results database of the National Library of Medicine, National Institutes of Health, USA, where Rebecca Williams is Assistant Director, Tony Tse is Program Analyst and Deborah Zarin is Director. No competing interests declared for William Harlan.

Contributors: All of the authors were involved in the conception of the article. Rebecca Williams was involved in the acquisition of the data, and all of the authors were involved in the analysis of the data. William Harlan drafted the article. All of the authors critically revised the manuscript for important intellectual content and approved the final version submitted for publication.

Acknowledgements: The authors thank Annice Bergeris, Sarah Kornmeier and Rachel Machta for their assistance in data collection and summarization.

Funding: Financial support for this article was received from the Intramural Research Program of the National Institutes of Health, National Library of Medicine.

\section{REFERENCES}

1. Avorn J. In defense of pharmacoepidemiology - embracing the yin and yang of drug research. $N$ Engl J Med 2007;357:2219-21

2. Chanock SJ, Manolio T, Boehnke M, et al. Replicating genotype-phenotype associations. Nature 2007;447:655-60.

3. Dickersin K, Min YI, Meinert CL. Factors influencing publication of research results. Follow-up of applications submitted to two institutional review boards. JAMA 1992;267:374-8.

4. Easterbrook PJ, Berlin JA, Gopalan R, et al. Publication bias in clinical research. Lancet 1991;337:867-72.

5. Zarin DA, Tse T. Medicine. Moving toward transparency of clinical trials. Science 2008;319:1340-2.

6. Enhancement of the scientific process and transparency of observational epidemiology studies 24-25 September 2009, London [workshop report no. 18]. Brussels (Belgium) European Centre for Ecotoxicology and Toxicology of Chemicals; 2009. Available: http://members.ecetoc.org/Documents/Document/20100108131222-WR _18.pdf (accessed 2010 Apr. 13).

7. Loder E, Groves T, Macauley D. Registration of observational studies. BMJ 2010;340:c950.

8. Should protocols for observational research be registered? Lancet 2010;375:348.

9. Krleza-Jeric K, Chan AW, Dickersin K, et al. Principles for international registration of protocol information and results from human trials of health related interventions: Ottawa statement (part 1). BMJ 2005;330:956-8. 
PRISTIQ is indicated for the symptomatic relief of major depressive disorder. The short-term efficacy of PRISTIQ (dessenlafoxine succinote extended-release toblets) hos been demonstroted in placebo-controlled trials of un to 8 weeks. The most commonly observed adverse events associated with the use of PRISTIQ (at an incidence $\geq 5 \%$ and at least twice the rate of placebo) were nausea (22\%), dizziness (13\%), hyperhidrosis $(10 \%)$, constipation $(9 \%)$, and decreased appefite $(5 \%)$.

PRISTIQ is not indicated for use in children under the age of 18. PRISTIQ is contraindicated in patients taking monoamine oxidase inhibitors (MAOls, including linezolid, an antibioitic) or in patients who have token MAOls within the preceding 14 days due to risk of serious, sometimes fatal, drug interactions with selective serotonin reuptake inhibitor (SSRI) or serotonin norepinephrine reuptake inhibitor (SNRI) treatment or with other serotonergic drugs. These interactions have been associoted with symptoms that include tremor, myoclonus, diaphoresis, nousea, vomiting, flushing, dizziness, hyperthermia with features resembling neuroleptic malignant syndrome, seizures, rigidity, autonomic instability with possible rapid fluctuations of vital signs, and mental status changes that indude extreme agitation progressing to delirium and como. Based on the half-life of desvenlafoxine succinate, at least 7 days should be allowed after stopping desvenlafaxine succinate and before starting on MAOI.

PRISTIQ is contraindicated in patients demonstrating hypersensitivity to desvenlafaxine succinate extendedreleasse, venlofoxine hydrochloride or to any excipients in the desvenlataxine formulation. Concomitant use of PRISTQ with products containing venlafoxine is not recommended.

Recent analyses of placebo-controlled clinical trial sofety databases from selective serotonin reuptake inhibitors (SSRIs) and other newer antidepressants suggest that use of these drugs in patients under the age of 18 may be associated with behavioural and emotional changes. including an increased risk of suicide ideation and behaviour over that of placebo.

The small denominators in the clinical trial database, as well as the variability in placebo rates, prectude reliable conclusions on the relative safety profiles among the drugs in the class. There are clinical trial and post-markefing reports with SSPRls and other newer anitidepresssants, in both pediatritics and adults, of severe agitation-type events that include: akathisia, agitation, disinhibition, emotional lability, hostility, aggression and depersonalization. In some cases, the events occurred within several weeks of starting treatment.

Rigorous clinical monitoring for suicide ideation or other indicators of potential for suicide behaviour is advised in patients of all ages, especially when initiating therapy or during any change in dose or dosage regimen. This includes monitoring for agitation-type emotional and behavioural changes.

Patients currently taking PRISTIQ should NOT be discontinued abruptly, due to risk of discontinuation symptoms. At the fime that a medical decision is made to discontinue an SSRI or other newer antidepressant drug, a gradual reduction in the dose, rather than an abrupt cessation is recommended.

Reference: 1. Wyeth Canada. PRSSTIQ Product Monograph, Avgust 2009. Product Monograph available upon request.

Member $\quad$ PRISTIQ

(R) Wyeth, owner,

$\overparen{\text { PAAB }}$ now a part of Pfizer Inc.

\section{Wyeth}

(C) 2010 Wyeth Canada

Montreal, Canada H4R 1J6

\section{Count on

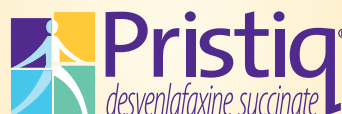

for powerful symptom relief 